\section{$\therefore$ \\ Heart Rate Variability and its Modulation Across Lifecycle Among Healthy Individuals}

IJCRR

Section: Healthcare ISI Impact Factor (2019-20): 1.628 IC Value (2019): 90.81 $\operatorname{SJIF}(2020)=7.893$

\section{Sharma Sowmya ${ }^{1}$, Thomas Tinku2 ${ }^{2}$, Sambashivaiah Sucharita ${ }^{1}$}

'Department of Physiology, St Johns Medical College, Bangalore, India; 'Department of Biostatistics, St Johns Research Institute, Bangalore, India.

\title{
ABSTRACT
}

Introduction: Autonomic imbalance is known to play a key role in health and disease. However, there is a lack of data exploring the sympathovagal balance across the lifecycle among the healthy Asian Indians

Objective: To compare the changes in HRV (Heart rate variability) indices among clusters of the healthy population across the life cycle.

Methods: Analysis was performed on pooled data categorised into 4 study groups including fetal ( $n=90)$, child ( $n=90)$, young adult $(n=134)$, and old ( $n=85)$. ECG was collected and subjected to HRV power spectral analysis.

Results: There was a significant difference in all log-transformed HRV indices between the study groups $(p<0.01)$. Low frequency (LF) and high frequency (HF) HRV indices were lower in the fetal and the older adult group compared to the child and young adult $(p<0.01)$. Effect size (Cohen's d) estimates for fetal and young adult groups for LF was 2.9 and that for HF was higher at 3.4. The effect size between young adult and old groups was 1.7 for LF and 2.1 for HF

Conclusion: There was an increment in HRV indices reflecting the maturity of cardiac vagal and cardiac sympathetic control of the heart from fetal to adult life. The degree of modulation across age groups was greater for the parasympathetic compared to the sympathetic component.

Key Words: Ageing, Heart rate variability, Lifecycle, Sympathovagal balance, Healthy, Autonomic imbalance

\section{INTRODUCTION}

The "sympathovagal balance" reflects the state of the autonomic nervous system which results from the sympathetic and parasympathetic influences. ${ }^{1}$ Though physiological modulations of sympathovagal balance are known to occur throughout the life of an individual especially with ageing, it is often studied in isolation. Longitudinal assessment of sympathovagal balance across the lifecycle is not feasible. Therefore, the next best approach involving cross-sectional studies have been attempted. ${ }^{2,3}$ There are pockets of longitudinal studies exploring sympathovagal balance among healthy individuals with a focus on middle-aged and elderly populations ${ }^{4}$ However, there is a lack of data especially from fetal, childhood phases and across the life cycle.

Sympathovagal imbalance (SVI) between the sympathetic and parasympathetic nervous systems has emerged as one of the key pathophysiological mechanisms by which one could explain the role of the autonomic nervous system in various clinical disorders. ${ }^{5,6}$ This is relevant especially among Asian Indians in whom reports of cardiovascular disease and metabolic syndrome are on the rise. South Asians including Indian's makeup one-quarter of the world's population and are at a greater risk of developing the chronic disease at a much younger age. ${ }^{7}$ Asian Indian phenotypes differ metabolically, with the majority developing chronic disease even at a normal body weight called "metabolically obese". The presence of ectopic fat along with reduced muscle mass/ function makes their body composition different from other populations. ${ }^{8} 9$ It will be interesting to explore the changes in a sympathovagal balance associated with age and body mass index (BMI) across the life cycle among the healthy Asian Indian population. This will help delineate the sympathovagal changes between healthy and disease states. Therefore, the current study is the first of its kind from healthy Asian

\section{Corresponding Author:}

Dr. Sambashivaiah Sucharita, MD, PhD, Professor, Department of Physiology, St John's Medical College, Bangalore-560034, India. Tel : +91 80 49466324; Fax: +91 80 25501088; Email: sucharita@stjohns.in

ISSN: 2231-2196 (Print)

Received: 23.01 .2021
ISSN: 0975-5241 (Online)

Revised: 15.03 .2021
Accepted: 09.05.2021 
Indians exploring the physiological changes across the life cycle ie., fetal to old age.

Heart rate variability (HRV) is a non-invasive technique widely used to quantify modulations in sympathetic and parasympathetic branches of the autonomic nervous system. ${ }^{10}$ Of late the advent of fetal HRV has allowed the exploration of autonomic modulation during fetal life as well. ${ }^{11}$ The impact of environmental factors during fetal life contributing to the development of chronic disease during adulthood needs to be explored. While a large body of literature using animal models have suggested the role of sympathetic nervous system activation to partially explain this phenomenon, ${ }^{12}$ this remains to be studied among humans. The study aimed to compare the changes in HRV indices among clusters of the healthy population of various ages across the life cycle i.e. from fetus to old age. Also, the study aimed to understand if body mass index plays a role in modulating age-related changes in HRV indices in each of the study groups.

\section{Methodology}

Data collected from 4 studies on various age groups of healthy populations at the Department of Physiology were used for the present analysis. The data collected include general characteristics, anthropometric parameters, and HRV indices derived using power spectral analysis. All participants provided written informed consent to take part in the studies which were approved by the Institution Ethics Review Board( IERB study ref Nos 113/2010, 168/2013,1/44/07). The baseline data was used for the current analysis from the data set which included healthy individuals to look at various physiological perturbations on heart rate variability indices. Details of the study groups including their recruitment details are as follows:

\section{Fetal Group:}

Data from 90 healthy women with a singleton pregnancy in their 3rd trimester were used for the present study. The participants were recruited from the Obstetrics outpatient department. The mothers were screened for the following inclusion and exclusion criteria: women with multiple pregnancies, those with chronic diseases like diabetes and hypertension were excluded from the study. Blood pressure measurements were performed to rule out orthostatic intolerance and gestational hypertension. An oral glucose challenge test was also performed to rule out gestational diabetes mellitus. Raw abdominal ECGs for maternal and fetal HRV indices and anthropometric details like the height and weight of the mother were collected on the same day of the visit.

\section{Child group:}

Data from 90 healthy children between the age of 3 to 8 years was collected from an ongoing birth cohort. The HRV was performed on children after parental consent and assent. A general physical examination was performed by a paediatrician to rule out any ailments present or past. The anthropometric measurement of height in meters and weight in kilograms which was used to calculate the body mass index $(\mathrm{kg} /$ $\mathrm{m}^{2}$ ) and ECG for HRV indices were collected on the same day of the visit.

\section{Young adult and Older Adult group:}

Data on heart rate variability and anthropometry of 134 young adult males between $20-40$ years and 85 older adults above 60 years were analyzed. As part of the recruitment process, participants were screened for chronic diseases (diabetes or hypertension), any form of anaemia, cancer, chronic infection including tuberculosis and neuropathy. None of the subjects reported any symptoms suggestive of peripheral and autonomic neuropathy. Lead II ECG and anthropometry was recorded. The institutional ethical board had approved the research study

\section{Assessment of heart rate variability:}

For collecting fetal ECGs, subjects were instrumented for the recording of both maternal and fetal ECG (Monica DK, UK) by placing pre-gelled disposable silver electrodes (Ambu blue sensor, Copenhagen, Denmark) on the maternal abdomen. After instrumentation and a mandatory 30-minute rest period, continuous abdominal ECG was obtained for 10 minutes in a quiet room in the supine position. Subjects were asked to avoid unnecessary movements during this period. ECG was collected using a sampling frequency of $900 \mathrm{~Hz}$ using an IBM compatible PC and a data acquisition package (Monica, UK). The data acquisition system collects raw ECG from which both maternal and fetal ECG were extracted. The same has been validated. ${ }^{13}$ The system also includes a threshold peak detection system, from which RR intervals of both mother and fetus were derived and used for power spectral analysis. ${ }^{14}$

In the child, young adult, and old group, lead II ECG was recorded following instrumentation. Following rest in supine posture for 30 minutes, measurements were performed for 10 minutes. Details of the signal processing and mathematical calculations have been discussed earlier. ${ }^{15}$ Briefly, spectral analysis was performed using a Fast Fourier Transform. The frequency resolution was 0.0078 and the highest frequency evaluated was $0.4 \mathrm{~Hz}$. The spectra obtained were averaged and power was calculated in two bands. The low-frequency band which is believed to reflect, predominantly the sympathetic nerve activity to the heart was calculated between $0.04-0.15 \mathrm{~Hz}$ band of RR power. The high-frequency band which is believed to reflect parasympathetic nerve activity to the heart was calculated from $0.15-0.4 \mathrm{~Hz}$. Along with absolute power, HRV indices were also calculated as normalised units where the power in the low and high-frequency bands 
is expressed as a percentage of the total power minus the power of the very-low-frequency band $(0.0-0.04 \mathrm{~Hz}){ }^{16}$

\section{Statistical analysis:}

The normality of the data was examined using the Kolmogorov-Smirnov test. The data is represented as mean and standard deviation when normally distributed and median (Interquartile range) when not normally distributed. As the HRV indices were not normally distributed, log-transformed data were used for further analysis. One-way ANOVA/ Kruskal Wallis test was used to analyze the effect of age groups on HRV indices. Post-hoc Bonferroni's test was used to examine pair-wise differences between age groups. The effect of weight/BMI in the association between age group HRV was examined using multiple linear regression of log-transformed HRV indices. Effect size (Cohen's d) comparison between the study groups in the post hoc analysis was performed to compare the pattern of changes in HRV indices between the groups. Statistical significance was considered at $p \leq 0.05$. The interaction effect of BMI and groups was examined in regression analysis of log-transformed HRV indices and statistical significance of interaction was considered at $p<0.1$.

\section{RESULTS}

The descriptive characteristics of the study groups are represented in Table 1. All the subject characteristics namely age, weight, height, BMI, waist circumference and heart rates were significantly different $(\mathrm{p}<0.05)$ between the study groups. On performing post hoc analysis, weight was significantly lower in the older adult group and child group compared to the young adult group. Similarly, height and BMI was lower in the child group compared to the young adult group. Height and BMI were comparable between young adult and older adult groups. Waist circumference was significantly higher in the older adult group and significantly lower in the child group compared to the young adult group. Heart rate was also significantly higher in the child group compared to the young adult group. Heart rates were comparable between the young adult group and the older adult group. Waist Median (Inter Quartile) ranges for HRV indices in absolute units $\left(\mathrm{msec}^{2}\right)$ in each of the groups are as follows. The LF power spectra were for fetal group [54.5(33-140)], child group [834.3(388.81329.1)], young adult [876.1(482.0-145.7)], and older adult group was [169.1(88.3-318.10)].For HF power spectra were for fetal group [ 31.0 (16-61)], child group [734.2 (318.71641.7)], young adult [ $874.2(502.9-1555.7)]$, and older adult group was [145.7 (67.2-207.3)]. For total power power spectra were for fetal group [317(143.5-527.3)], child group [2039.0(1025.9-3641.4)], young adult [2567.8(1558.54674.9)] and older adult group was [576.3(348.5-1134.7)]. Figure 1 represents the log-transformed HRV indices among all the study groups. There was a significant difference in all log-transformed indices between the study groups $(\mathrm{p}<0.01)$. LF and HF HRV indices were significantly lower in the fetal ( $4.2 \pm 1.0$ for LF, $3.4 \pm 0.9$ for HF ) and the older adult group (5.2 \pm 0.9 for LF, $4.8 \pm 0.9$ for HF) compared to the child (6.6 \pm 0.8 for $L F, 6.6 \pm 1.1$ for HF) and young adult $(6.7 \pm 0.9$ for LF, $6.8 \pm 1.0$ for HF) $(p<0.01)$. The young adults and child groups had comparable HRV. The magnitude of increment and decrement in LF and HF across the study groups was calculated concerning the young adult group. The effect size between the fetal and young adult group for LF was 2.9 and that for HF was higher at 3.4. The effect size between young adult and old groups was 1.7 for LF and 2.1 for HF. On comparing the LF/HF ratio, the fetal group had a significantly higher ratio compared to the three study groups (Figure 2). Analysis of LF and HF normalized units $\mathrm{(nu})$ also demonstrated similar results. There was a positive association between log HF and age in the child group and a negative association in young adult and older groups after controlling for BMI (Table 2). There was a significant interaction $(p=0.064)$ between BMI and group for log HF such that the regression coefficient for log HF on BMI in the elderly was significantly lower compared to that of young adults. There was no association between gestational age and HRV indices in the fetal group.

\section{DISCUSSION}

The present study demonstrated a significant difference in the heart rate variability indices among the study groups. There was a significant increase in all the HRV indices in the child and adult groups when compared to the fetal group. Also, there was a significant decrease in all the HRV indices in the older adult group when compared to the young adult group. Low frequency to high-frequency ratio, a measure of sympathovagal balance was significantly higher in the fetal and older adult group when compared to young adult and child groups.

The present study for the first time explored a lifecycle approach with a specific focus on modulations in HRV indices to study sympathovagal balance. Autonomic modulations play an essential role in maintaining normal physiological functions throughout the human lifecycle. ${ }^{17}$ With the invention of fetal ECG extraction from maternal ECG, fetal autonomic regulation evaluation has become a possibility. Studies use fetal heart rate and their pattern in terms of accelerations and decelerations to predict fetal wellbeing. ${ }^{18}$ However, fetal heart rate variability based studies using spectral analysis are limited. Autonomic maturation during pregnancy can be partly explained by the polyvagal theory which states that from the time of conception, the first autonomic branch to develop is the unmyelinated, primitive vagal nerve. This is followed by the development of the sympathetic nervous system and this is closely linked to gestational age during 
early pregnancy. ${ }^{19}$ Hence during the early fetal period, variability in sympathetic tone is said to play a role in heart rate and blood pressure variability. ${ }^{20}$ The parasympathetic division of the ANS gradually develops during late pregnancy and continues following birth. ${ }^{21}$ The data from the present study demonstrated that the low frequency/high-frequency ratio an indicator of sympathovagal balance was highest among the fetal group compared to child, adult, and old study groups. This points towards the fact that during fetal life there is sympathetic predominance. In an Indian context, this might be relevant as epigenetic changes are believed to be the basis of the change in programming phenotype. The role of in utero environment especially maternal nutritional status (Iron, Vitamin B12, and Zinc) could play a key role in autonomic maturation. ${ }^{22}$ The role of fetal sympathetic and vagal maturation and maternal nutritional status needs to be further explored.

Studies have demonstrated that an increase in the total number of myelinated vagal fibres from fetal life is a gradual process and extends until the child reaches adolescence. ${ }^{21}$ This is reflected even in our data which showed a significant increment in HRV indices in the child group and adult group when compared to fetal data. Apart from ageing, another important physiological factor affecting HRV is the change in body composition. ${ }^{23}$ There was a significant and positive correlation between age and HRV indices in the child group even after controlling for BMI. Moreover, the data suggested increment in high frequency to be greater compared to low frequency. This could be due to an increase in myelinated vagal fibres which is required for not only improved visceral regulation but also for behavioural regulation which would support social engagement behaviours following birth. ${ }^{24}$

Among the adult population, the ageing-associated decline in HRV indices demonstrated in earlier studies was also noted as part of the current study. ${ }^{4,25}$ The decline in high frequency was greater compared to low frequency. The possible mechanisms for underlying changes in parasympathetic modulation with ageing could be alterations in cholinergic and muscarinic pathways. The cardiac acetylcholine release response to stimulation decreases with ageing including muscarinic receptor activity and density. ${ }^{26}$ This loss of protective vagal reflexes may impair the functional capacity to respond to various stimuli with ageing and could be one of the potential reasons for increased vulnerability in developing chronic diseases with ageing. ${ }^{27}$ Our previous studies have linked decreased autonomic function predominantly parasympathetic nervous activity to visceral fat among lean or normal BMI healthy adults. ${ }^{28}$ Even in the present study, the high frequency was negatively and significantly associated with BMI in both the young and older adult groups. However, the significant negative association between age and high-frequency components of HRV indices in both young adult and older groups persisted even after controlling for BMI. A study of interactions between BMI across age groups showed the effect of BMI on high frequency was significantly lower in older adults compared to younger adults. Few previous studies have reported improved survival among subjects with elevated BMI among the elderly. ${ }^{29,30}$ The deleterious effect of being overweight or underweight on mortality was mainly observed in young and middle-aged subjects rather than very aged subjects. Obesity in the young and middle-aged is associated with increased cardiac risk like hypertension, diabetes mellitus and hyperlipidemia. ${ }^{31}$ One of the proposed reasons for the improved survival among the elderly was modulations of the inflammatory response and increased nutritional and metabolic reserve over prolonged exposure. ${ }^{32}$ Also the other reason could be that the study subjects selected in the older adult group may reflect selection bias where younger obese individuals who progress into old age with cardiovascular disorders were excluded. Thus overweight/underweight subjects who survived cardiovascular disease and other illnesses earlier in life may reflect genetic predisposition which may have protected them from increased cardiovascular risk in old age as well. Thus the lower effect of BMI on autonomic modulations seen in the older adult group could be a combination of genetic factors along with adequate energy stores in the form of fat that may protect these individuals from catabolic processes. These findings are important especially for a country like India which has experienced a demographic transition in the past few decades, causing almost a tripling of the population over the age of 60 years. ${ }^{33}$ With the added burden of malnutrition (both macro and micronutrient deficiency) among the elderly, there is a need for evidence-based clinical trials to plan public health strategies in India. With more than $30 \%$ of the Indian population considered to have BMI in the range of low to normal and approximately the same percentage being overweight and obese ${ }^{34}$ the current data becomes relevant in the context that this might also help future studies exploring sympathovagal balance among Indians with various clinical conditions. A simpler technique like HRV could play a key role and go a long way to understand the impact of such interventions.

One of the key strengths of the study was that it was performed on a healthy population across the human life cycle. However, lacunae of the study included the inability to explore the role of factors including percentage body fat, lean mass, physical activity pattern, dietary intakes, and stress response across the lifecycle. Further, studies are warranted to explore physiological perturbation on autonomic modulation across the lifecycle.

\section{CONCLUSION}

HRV indices across the lifecycle reflect the evolving modulations of the cardiac vagal and cardiac sympathetic nervous system from fetal to adult life. The sympathovagal balance 
modulation was highest among the fetal group compared to the three study groups with greater modulations in parasympathetic component compared to sympathetic component across age groups. Also, BMI plays a role in modifying sympathovagal balance across age groups but the effect significantly decreases with ageing

\section{ACKNOWLEDGEMENT}

The authors would like to thank Dr. Dwarkanath P for helping with the collection of data of the child group. We are also thankful to Dr. Benny and Mr. Ravikanth for helping us with young and elderly group data collection and analysis. We are thankful to Mrs. Martha Monica and Mrs. Nusrat Fathima for helping in the collection of fetal data.

The authors also acknowledge the immense help received from the scholars whose articles are cited and included in references of this manuscript. The authors are also grateful to authors/editors/publishers of all those articles, journals and books from where the literature for this article has been reviewed and discussed.

Conflict of interest: The authors declare that there are no conflicts of interest

Source of Funding: We thank the Research Society, St John's Medical College, for funding this study partially.

\section{Authors' Contribution}

Role of each author in the work: SSH and SS hypothesized, analysed and interpreted the study. SSH wrote the initial draft of the manuscript. TT provided statistical guidance in data analyses. All co-authors participated in manuscript preparation and critically reviewed all sections of the text for important intellectual content.

\section{REFERENCES}

1. Goldberger JJ. Sympathovagal balance: how should we measure it? Am J Physiol. 1999 Apr; 276(4): H1273-80.

2. Longin E, Dimitriadis C, Shazi S, Gerstner T, Lenz T, König S et al. Autonomic nervous system function in infants and adolescents: impact of autonomic tests on heart rate variability. Pediatr Cardiol. 2009 Apr; 30(3):311-24.

3. Parashar R, Amir M, Pakhare A, Rathi P, Chaudhary L. Age-Related Changes in Autonomic Functions. J Clin Diagn Res. 2016 Mar;10(3): CC11-15.

4. Jandackova VK, Scholes S, Britton A, Steptoe A. Are Changes in Heart Rate Variability in Middle-Aged and Older People Normative or Caused by Pathological Conditions? Findings From a Large Population-Based Longitudinal Cohort Study. J Am Heart Assoc. 2016 Feb12;5(2):e002365.

5. Fleischer J. Diabetic autonomic imbalance and glycemic variability. J Diabetes Sci Technol. 2012 Sep1;6(5):1207-15.

6. Pal GK, Adithan C, Amudharaj D, Dutta TK, Pal P, Nandan $\mathrm{PG}$, et al. Assessment of sympathovagal imbalance by spectral analysis of heart rate variability in prehypertensive and hypertensive patients in Indian population. Clin Exp Hypertens. 2011;33(7):478-83.

7. Prasad DS, Kabir Z, Dash AK, Das BC. Prevalence and risk factors for metabolic syndrome in Asian Indians: A community study from urban Eastern India. J Cardiovasc Dis Res. 2012 Jul;3(3):204-11.

8. Banerji MA, Faridi N, Atluri R, Chaiken RL, Lebovitz HE. Body composition, visceral fat, leptin, and insulin resistance in Asian Indian men. J Clin Endocrinol Metab. 1999;84(1):137-44.

9. Sucharita S, Pranathi R, Correa M, Keerthana P, Ramesh LJ, Bantwal G, et al. Evidence of higher intramyocellular fat among normal and overweight Indians with prediabetes. Eur J Clin Nutr. 2019; 73:1373-81.

10. Shaffer F, Ginsberg JP. An Overview of Heart Rate Variability Metrics and Norms. Front Public Health. 2017Sep; 5:258.

11. Schneider U, Bode F, Schmidt A, Nowack S, Rudolph A, Doelcker E, et al. Developmental milestones of the autonomic nervous system revealed via longitudinal monitoring of fetal heart rate variability. PLOS ONE. 2018; 13(7): e0200799.

12. Rahmouni K. Sympathetic tone in the young: the mother weighs in. Hypertension. $2010 \mathrm{Jan} ; 55(1): 21-22$.

13. Cohen WR, Ommani S, Hassan S, Mirza FG, Solomon M, Brown R, et al. Accuracy and reliability of fetal heart rate monitoring using maternal abdominal surface electrodes. Acta Obstet Gynecol Scand. 2012 Nov; 91(11):1306-13.

14. Montalvo-Jaramillo CI, Pliego-Carrillo AC, Peña-Castillo MÁ, Echeverria JC, Becerril-Villanueva E, Pavon L, et al. Comparison of fetal heart rate variability by symbolic dynamics at the third trimester of pregnancy and low-risk parturition. Heliyon. 2020 Mar 12;6(3):e03485.

15. Sucharita S, Bharathi AV, Kurpad AV, Vaz M. A comparative study of tests of cardiac parasympathetic nervous activity in healthy human subjects. Physiol Meas. 2002; 23 (2): 347-54.

16. Heart rate variability: standards of measurement, physiological interpretation and clinical use. Task Force of the European Society of Cardiology and the North American Society of Pacing and Electrophysiology. Circulation. 1996;93(5):1043-65.

17. McCorry LK. Physiology of the autonomic nervous system. Am J Pharm Educ. 2007 Aug 15;71(4):78.

18. Lee CY, Di Loreto PC, O'Lane JM. A study of fetal heart rate acceleration patterns. Obstet Gynecol. 1975;45(2):142-46.

19. Porges SW. Orienting in a defensive world: mammalian modifications of our evolutionary heritage. A Polyvagal Theory. Psychophysiology. 1995;32(4):301-18.

20. Segar JL. Ontogeny of the arterial and cardiopulmonary baroreflex during fetal and postnatal life. Am J Physiol. 1997; 273:R457-71.

21. Sachis PN, Armstrong DL, Becker LE, Bryan AC. Myelination of the human vagus nerve from 24 weeks postconceptional age to adolescence. J Neuropathol Exp Neurol. 1982;41:466-72.

22. Gernand AD, Schulze KJ, Stewart CP, West KP Jr, Christian P. Micronutrient deficiencies in pregnancy worldwide: health effects and prevention. Nat Rev Endocrinol. 2016 May;12(5):27489.

23. Triggiani AI, Valenzano A, Ciliberti MAP, Moscatelli F, Villani $\mathrm{S}$, Monda $\mathrm{M}$, et al. Heart rate variability is reduced in underweight and overweight healthy adult women. Clin Physiol Funct Imaging. 2017; 37: 162-67.

24. Porges SW, Furman SA. The Early Development of the Autonomic Nervous System Provides a Neural Platform for Social Behavior: A Polyvagal Perspective. Infant Child Dev. 2011; 20:106-18. 
25. Jindal RD, Vasko RC Jr, Jennings JR, Fasiczka AL, Thase ME, Reynolds CF $3^{\text {rd }}$ et al. Heart rate variability in depressed elderly. Am J Geriatr Psychiatry. 2008 Nov;16(11):861-66.

26. Brodde OE, Konschak U, Becker K, Ruter F, Poller U, Jakubetz $\mathrm{J}$ et al. Cardiac muscarinic receptors decrease with age. In vitro and in vivo studies. J Clin Invest. 1998 Jan 15;101(2):471-78.

27. Vaseghi M, Shivkumar K. The role of the autonomic nervous system in sudden cardiac death. Prog Cardiovasc Dis. 2008 May-Jun; 50(6):404-19.

28. Sowmya S, Thomas T, Bharathi AV, Sucharita S. A body shape index and heart rate variability in healthy Indians with low body mass index. J Nutr Metab. 2014; 2014:865313.

29. Weiss A, Beloosesky Y, Boaz M, Yalov A, Kornowski R, Grossman E. Body mass index is inversely related to mortality in elderly subjects. J Gen Int Med. 2008; 23(1):19-24.

30. Dahl AK, Fauth EB, Ernsth-Bravell M, Hassing LB, Ram N, Gerstof $\mathrm{D}$ et al. Body mass index, change in body mass index, and survival in old and very old persons. J Am Geriat Soc. 2013; 61(4): 512-18.
31. Wannamethee SG, Shaper AG, Walker M. Overweight and obesity and weight change in middle-aged men: impact on cardiovascular disease and diabetes. J Epidemiol Community Health 2005;59: 134-39.

32. Horwich TB, Fonarow GC. The impact of obesity on survival in patients with heart failure. Heart Failure Monitor. 2002; 3(1): 8-14.

33. Dey S, Nambiar D, Lakshmi JK, Sheikh K, Reddy KS. The health of the Elderly in India: Challenges of Access and Affordability. In: National Research Council (US) Panel on Policy Research and Data Needs to Meet the Challenge of Aging in Asia; Smith JP, Majmundar M, editors. Ageing in Asia: Findings From New and Emerging Data Initiatives. Washington (DC): National Academies Press (US),2012;15.

34. Indian Institute of Population Sciences, Mumbai, India, National Family Health Survey-3 2006; 1: 303-313.

\section{Table 1: Subject characteristics across four study groups}

\begin{tabular}{|c|c|c|c|c|}
\hline Characteristics & $\begin{array}{c}\text { Fetal group (Maternal } \\
\text { characteristics) } \\
\mathrm{N}=90 \\
\text { Mean } \pm \mathrm{SD}\end{array}$ & $\begin{array}{l}\text { Child group } \\
\qquad \begin{array}{l}\mathrm{N}=90 \\
\text { Mean } \pm \mathrm{SD}\end{array}\end{array}$ & $\begin{array}{l}\text { Young adult group } \\
\qquad \begin{array}{c}\mathrm{N}=134 \\
\text { Mean } \pm \text { SD }\end{array}\end{array}$ & $\begin{array}{c}\text { Older adult group } \\
\begin{array}{c}\mathrm{N}=\mathbf{8 5} \\
\text { Mean } \pm \text { SD }\end{array}\end{array}$ \\
\hline Age (Years) & $\begin{array}{c}35.8 \pm 2.5 \text { (gestational age } \\
\text { in weeks) }\end{array}$ & $5 \cdot 7 \pm 1.4$ & $24.8 \pm 8.4$ & $66.1 \pm 6.5^{*}$ \\
\hline Weight (Kg) & $67.4 \pm 12.7$ & $17.8 \pm 4.6$ & $56.6 \pm 9 \cdot 3$ & $52.1 \pm 8.3^{*}$ \\
\hline Height (m) & $1.54 \pm 0.1$ & $1.11 \pm 0.1$ & $1.7 \pm 0.1$ & $1.6 \pm 0.1^{*}$ \\
\hline BMI $\left(\mathrm{Kg} / \mathrm{m}^{2}\right)$ & & $14 \cdot 3 \pm 2.1$ & $20.2 \pm 2.7$ & $20.2 \pm 2.5^{*}$ \\
\hline Waist circumference (m) & & $48.3 \pm 4.6$ & $71.8 \pm 8.0$ & $77 \cdot 3 \pm 9.0^{*}$ \\
\hline Hip circumference (m) & & $55.2 \pm 5 \cdot 7$ & $86.6 \pm 7.1$ & $86.2 \pm 5.8^{*}$ \\
\hline Waist hip ratio & & $0.9 \pm 0.0$ & $0.8 \pm 0.0$ & $0.9 \pm 0.1$ \\
\hline Heart rate (bpm) & $143 \cdot 3 \pm 10.2$ & $96.0 \pm 11.2$ & $64.0 \pm 8.5$ & $64.6 \pm 12.8^{*}$ \\
\hline
\end{tabular}

${ }^{*} \mathrm{p}<0.05$ on ANOVA, BMI- Body Mass Index

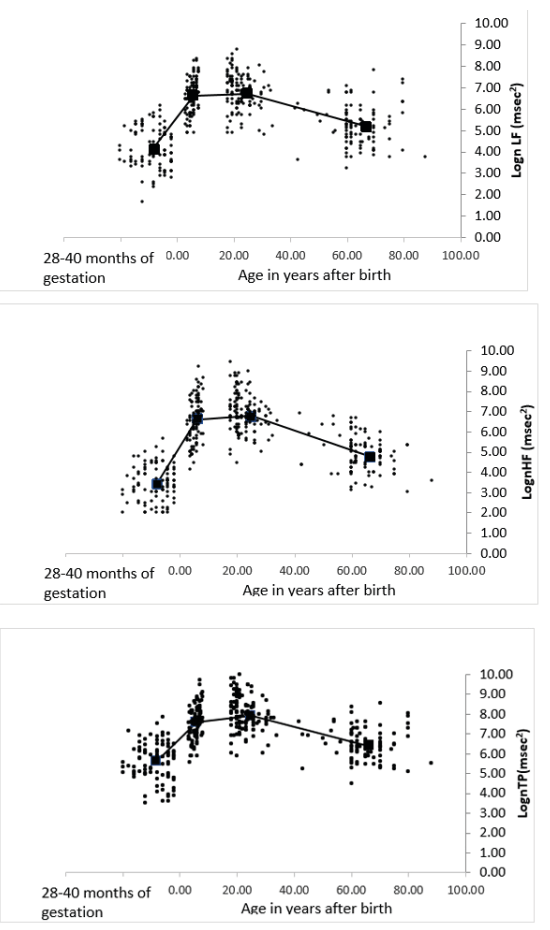

Figure 1: Heart rate variability indices across study groups 


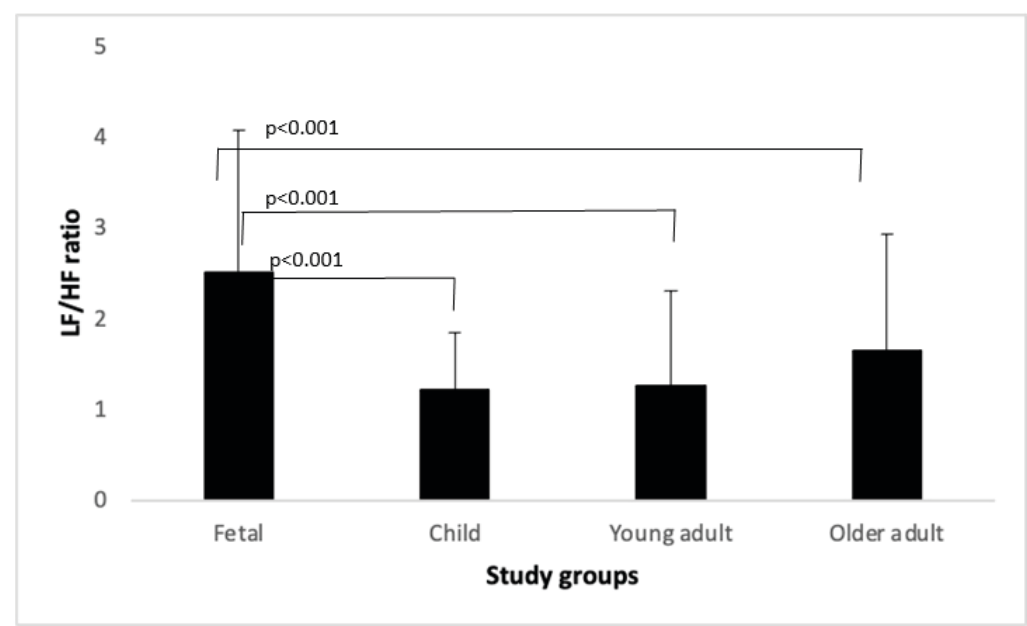

Figure 2: Comparison of sympathovagal balance (Low Frequency/High-Frequency ratio) across age groups. $\mathrm{P}<0.05$ between the groups using ANOVA.

$p<0.05$ on One-way ANOVA. Fetal group was significantly higher compared to child, young adult, and older adult group

Table 2: Linear regression model to understand the effect of age and body composition on HRV indices and sympathovagal balance

\begin{tabular}{|c|c|c|c|c|c|}
\hline Groups & Linear Regression Model & Variable & $\begin{array}{c}\operatorname{Logn} \mathrm{LF} \\
\beta(\operatorname{Se} \beta)\end{array}$ & $\begin{array}{c}\operatorname{Logn} H F \\
\beta(\operatorname{Se} \beta)\end{array}$ & $\begin{array}{c}\text { LF/HF ratio } \\
\beta(\mathrm{Se} \beta)\end{array}$ \\
\hline \multirow{3}{*}{ Fetal } & Model 1 & Gestational Age & $-0.03(0.04)$ & $0.01(0.04)$ & -0.10 (o.06) \\
\hline & \multirow[t]{2}{*}{ Model 2} & Gestational age & $-0.03(0.04)$ & $0.02(0.04)$ & $-0.11(0.07)$ \\
\hline & & Maternal Weight & $-0.00(0.01)$ & $-0.01(0.00)$ & $-0.00(0.01)$ \\
\hline \multirow[t]{3}{*}{ Child } & Model 1 & Age & $0.25(0.06) *$ & $0.36(0.07) *$ & $-0.10(0.05) *$ \\
\hline & \multirow[t]{2}{*}{ Model 2} & Age & $0.27(0.06) *$ & $0.36(0.07) *$ & $-0.11(0.05) *$ \\
\hline & & BMI & $-0.02(0.04)$ & $-0.03(0.05)$ & $-0.00(0.3)$ \\
\hline \multirow[t]{3}{*}{ Young } & Model 1 & Age & $-0.04(0.01) *$ & $-0.06(0.01) *$ & $0.03(0.01) *$ \\
\hline & \multirow[t]{2}{*}{ Model 2} & Age & $-0.06(0.01) *$ & $-0.07(0.01) *$ & $0.03(0.01) *$ \\
\hline & & BMI & $0.07(0.03) *$ & $0.10(0.03)$ * & $0.00(0.04)$ \\
\hline \multirow[t]{3}{*}{ Elderly } & Model 1 & Age & $0.01(0.02)$ & $-0.03(0.02) *$ & $0.02(0.02)$ \\
\hline & \multirow[t]{2}{*}{ Model 2} & Age & $0.01(0.02)$ & $-0.04(0.02) *$ & $0.02(0.02)$ \\
\hline & & BMI & $0.01(0.04)$ & $-0.08(0.04) *$ & $0.07(0.06)$ \\
\hline
\end{tabular}

${ }^{*} \mathrm{p}<0.05$ 